\title{
Sequential Finger-tapping Learning Mediated by the Primary Motor Cortex and Fronto-parietal Network: A Combined MRI-MRS Study
}

\section{Shuki Maruyama}

National Institute for Physiological Sciences

\section{Masaki Fukunaga}

National Institute for Physiological Sciences

Sho K. Sugawara

National Institute for Physiological Sciences

Yuki H. Hamano

National Institute for Physiological Sciences

Tetsuya Yamamoto

National Institute for Physiological Sciences

Norihiro Sadato ( $\nabla$ sadato@nips.ac.jp )

Department of System Neuroscience, Division of Cerebral Integration, National Institute for Physiological Sciences (NIPS), 38 Nishigonaka, Myodaiji, Okazaki, Aichi 444-8585, Japan

\section{Research Article}

Keywords: primary motor cortex (M1), task functional magnetic resonance imaging, sensorimotor network

Posted Date: February 17th, 2021

DOI: https://doi.org/10.21203/rs.3.rs-197014/v1

License: (a) (1) This work is licensed under a Creative Commons Attribution 4.0 International License. Read Full License 


\section{Abstract}

The primary motor cortex (M1) is crucial for motor learning. However, the interaction of the M1 with other brain areas during motor learning remains unclear. We hypothesized that the fronto-parietal execution network (FPN) provides the learning-related information that is crucial for flexible cognitive control required for practice. We assessed the network-level changes during sequential finger-tapping learning "as fast and as accurately as possible", by combining magnetic resonance spectroscopy, task functional magnetic resonance imaging (fMRI), and resting-state fMRI methods using a 7T MR machine. An increase in the glutamate/GABA ratio in the right M1 was positively correlated with task performance improvement. There was a motor learning-related increase in preparatory activity in the fronto-parietal region, with an overlap between the FPN and sensorimotor network (SMN). The learning-related increments in M1-seeded functional connectivity with the FPN, but not the SMN, were positively correlated with changes in the glutamate/GABA ratio in M1. These connectivity changes were more prominent in the parietal region than in the frontal region. Our findings indicate that motor learning driven by cognitive control is associated with local variation in the excitatory-inhibitory balance in the M1 that reflects remote connectivity with the FPN, thereby representing the formation of declarative procedural skills.

\section{Introduction}

Motor learning refers to the acquisition of new spatiotemporal muscle activation patterns ${ }^{1}$. Practice is a critical factor for motor learning, which is characterized by a goal-seeking process with feedback, leading to a configurational change in movement in terms of speed and accuracy ${ }^{2}$, representing the performer's attempt to reach a goal ${ }^{3,4}$. Thus, practice requires externally directed attention toward a goal and feedback, and internally directed attention focused on motor control. The difference between a goal and feedback is referred to as a challenge, which is crucial for motor skill learning and retention ${ }^{4,5}$. Challenge makes trainees exert an effort into the relevant training; for example, the sequential finger-tapping learning paradigm is frequently utilized in the neuroimaging field ${ }^{6-11}$ wherein participants are usually instructed to practice a given sequence "as fast and as accurately as possible." In these situations, participants must first retrieve the sequence to conduct the practice. Speed pressure enhances the learning process because the instruction of "as fast as possible" maintains the difference between the goal and performed output; thus, the task remains challenging. In addition to motor control, this type of practice for motor learning requires flexible cognitive control ${ }^{12}$ of externally and internally directed attention.

The primary motor cortex (M1) plays an essential role in motor skills learning ${ }^{1,13-15}$. M1 consists of the intrinsic horizontal connection network necessary to support learning-induced reorganization ${ }^{1}$ dependent on the precise balance of excitatory and inhibitory signaling within the system. Control at the local inhibitory level is critical to enable the functional restructuring of intracortical connections, leading to a change in the M1 output map ${ }^{1}$. The modulation of inhibitory GABA levels in M1 by anodal transcranial direct current stimulation, as measured by magnetic resonance spectroscopy (MRS), enhances motor 
learning ${ }^{16}$. Kolasinski et al. ${ }^{17}$ reported a dynamic reduction of GABA levels within M1 during motor skill learning using a serial reaction time task and MRS. Combining MRS and resting-state functional magnetic resonance imaging ( $\mathrm{fMRI}$ ) has permitted exploration of the relationship between learningrelated changes in the resting-state network and the formation of motor engrams in M1. A learningrelated reduction in GABA levels in $\mathrm{M} 1$ correlated with functional connectivity strength changes in the resting-state sensorimotor network (SMN) in long-term motor learning ${ }^{18}$. Baseline GABA levels in M1 are positively correlated with motor learning-related changes in resting-state functional connectivity between the bilateral M1s and between the right M1 and left superior parietal cortex ${ }^{19}$. These studies evaluated the relationship between GABA levels of the $\mathrm{M} 1$ and network changes in motor task-relevant regions.

Meanwhile, as a goal-seeking behavior, practice for motor learning requires executive control, suggesting the involvement of the fronto-parietal execution network (FPN) ${ }^{20}$. Herein, we hypothesized that learningrelated information during goal-seeking practice is provided by the FPN, in addition to the SMN. We measured the levels of glutamate (Glu) and GABA in the M1, as these neurotransmitters play an essential role in the neural circuits underpinning learning and memory ${ }^{21,22}$. We combined MRS, task fMRI, and resting-state $\mathrm{fMRI}$ to depict the network level changes during sequential finger-tapping learning with the non-dominant left hand, under speed-pressure, using a 7T MR machine (see Materials and Methods for details; Fig. 1). This study aimed to depict the M1-centered network for motor learning through goalseeking practice. To control the learning effect non-specific to the sequence, another group of 13 participants underwent the identical procedure, except that they tapped 120 different sequences.

\section{Results}

\section{MRS spectra}

Figure $2 A$ represents an example of MR spectra within M1 obtained using the 7T MR system. The $2 \times 2 \times 2$ $\mathrm{cm}^{3}$ volume of interest was centered over the hand knob area of the right M1 identified using $\mathrm{fMRI}$ during a finger opposition task (red), and was superimposed on T1w MPRAGE images. To assess whether the changes in metabolite concentrations were due to fluctuations in spectral quality, we evaluated the Cramer-Rao lower bounds (CRLB), linewidth, and SNR. MRS spectra provided reliable estimates of multiple metabolites, with a CRLB $<15 \%$. A repeated-measures ANOVA revealed no significant main effect of time (pre-task vs. during-task vs. post-task) on CRLB, linewidth, and SNR (Table 1).

\section{Table 1}

Magnetic resonance spectroscopy spectra quality of pre-task, during-task, and post-task periods 


\begin{tabular}{|lllll|}
\hline & CRLB (GABA) & CRLB (Glu) & CRLB (tCr) & Linewidth \\
\hline Pre-task & $9.7 \pm 1.1$ & $2.1 \pm 0.2$ & $1.9 \pm 0.2$ & $12.6 \pm 1.1$ \\
\hline During-task & $9.5 \pm 1.1$ & $2.1 \pm 0.3$ & $1.9 \pm 0.3$ & $12.7 \pm 1.3$ \\
\hline Post-task & $9.7 \pm 1.0$ & $2.2 \pm 0.4$ & $1.9 \pm 0.3$ & $12.8 \pm 1.3$ \\
$\begin{array}{l}\text { Main effect } \\
\text { of time }\end{array}$ & $\begin{array}{l}F[2,74]=0.984 \\
p=0.379\end{array}$ & $\begin{array}{l}F[2,74]=3.171 \\
p=0.059\end{array}$ & $\begin{array}{l}F[2,74]=0.196 \\
p=0.823\end{array}$ & $\begin{array}{l}F[2,74]=1.076 \\
p=0.346\end{array}$ \\
\hline
\end{tabular}

Data are presented as mean \pm standard deviation (SD) for $\mathrm{n}=38$.

CRLB, Cramer-Rao lower bounds; Glu, glutamate; $\mathrm{tCr}$, total creatine

Figure $2 \mathrm{~B}$ represents the distribution of the concentrations of $\mathrm{GABA} / \mathrm{tCr}$ and $\mathrm{Glu} / \mathrm{tCr}$ in the pre-, during-, and post-task periods. The variation in neurotransmitter concentration was analyzed using repeatedmeasures ANOVA with time as a factor (pre-task vs. during-task vs. post-task). No significant change in GABA/tCr concentration $\left(F_{(2,48)}=0.114 ; p=0.893\right)$ was observed. A significant main effect of time on Glu/tCr concentration $\left(F_{(2,48)}=11.857 ; p=6.536 \times 10^{-5}\right)$ was found. Post-hoc one-sample t-tests revealed significant reductions in the $\mathrm{Glu} / \mathrm{tCr}$ concentrations between pre- and post-task periods $\left(p=1.860 \times 10^{-4}\right.$ with Bonferroni correction) and between during and post-task periods $(p=0.040$ with Bonferroni correction).

\section{Task performance}

Task performance was evaluated using the transition time of the consecutive finger tapping (Fig. 3A). The transition times were $258.063 \pm 46.213$ for block $1,202.490 \pm 31.049$ for block 2, $184.320 \pm 26.285$ for block $3,178.237 \pm 22.600$ for block 4 , and $173.673 \pm 19.417$ for block 5 (mean \pm SD). Repeatedmeasures ANOVA revealed a significant main effect of time (blocks $1-5)\left(F_{(4,96)}=124.035 ; p=\right.$ $\left.4.872 \times 10^{-37}\right)$. Post-hoc one-sample t-tests revealed that the transition time did not significantly differ between blocks 4 and 5 ( $p=0.389$ with Bonferroni correction), indicating that performance plateaued. The relationship between the change in Glu/GABA ratio within M1 and performance improvement was evaluated using linear regression analysis. A positive correlation was observed between the change in the Glu/GABA ratio and performance improvement $\left(r_{(25)}=0.42, p=0.038\right)$ (Fig. 3B).

\section{Execution-related and preparation-related activity}

Task fMRI scans revealed the task execution-related activity in the bilateral M1, cerebellum (CB) lobules, supplementary motor area (SMA), thalamus (Thal), superior parietal lobule (SPL), and right primary somatosensory cortex (S1) family-wise error (FWE) corrected $p<0.05$ at voxel-level) (Fig. 4A). The preparation-related activity was observed in the bilateral putamen (Put), the insula, the M1, the SMA, the 
thalamus, the SPL, the middle occipital lobe, the right S1, and the middle frontal gyrus (MFG) (FWEcorrected $p<0.05$ at peak level; Fig. 4B).

\section{Linear increments in execution-related and preparation-related activity}

We observed linear increments in execution-related activity in the right $\mathrm{M} 1, \mathrm{~S} 1$, and the inferior occipital lobe with a lenient threshold (uncorrected $p<0.001$ at voxel-level and FWE-corrected $p<0.05$ at the cluster level; Fig. 5A). In contrast, linear increments in preparation-related activity were observed in the right M1 and S1, and the SMA. A linear increase in preparatory activity was also found in fronto-parietal regions, including the bilateral inferior parietal lobule (IPL), the MFG, STG, the thalamus, the CB lobules, the anterior cingulate cortex, and the middle cingulate cortex (FWE-corrected $p<0.05$ at the cluster level; Fig. 5B).

\section{Resting-state functional connectivity before and after motor learning}

The learning-related network, depicted as linear increments in preparation-related activity using task fMRI, overlapped with the FPN and SMN templates provided by the CONN toolbox (Fig. 6B). The relationships between Glu/GABA changes within M1 and resting-state M1 seed-based functional connectivity changes in the SMN and FPN after learning were investigated. A positive correlation was observed between changes in the Glu/GABA ratio and M1 seed-based resting-state functional connectivity changes in the FPN $\left(r_{(25)}=0.48, p=0.016\right)$; no correlation was observed in the SMN $\left(r_{(25)}=-0.16, p=0.435\right)$ (Fig. 6C). The correlation between the FPN and changes in the Glu/GABA ratio was more prominent in parietal regions than in frontal regions (lateral prefrontal cortex [LPFC], $r_{(25)}=0.35, p=0.087$; posterior parietal cortex [PPC], $\left.r_{(25)}=0.58, p=0.002\right)$ (Fig. 7).

\section{Sequence non-specific learning effect in the Control group}

The control group showed no significant change in GABA/tCr concentration $\left(F_{(2,24)}=0.275 ; p=0.762\right)$ and $\mathrm{Glu} / \mathrm{tCr}$ concentration $\left(F_{(2,24)}=3.014 ; p=0.068\right)$ in the right M1 (Fig. 8).

As demonstrated in Figure 9A, the transition times in non-specific learning were $466.960 \pm 97.564$ for block $1,409.301 \pm 77.179$ for block 2, $403.520 \pm 64.868$ for block $3,400.546 \pm 66.247$ for block 4 , and $393.746 \pm 64.553$ for block 5 (mean \pm SD). Although repeated-measures ANOVA revealed a significant main effect of time (blocks $1-5)\left(F_{(4,48)}=21.064 ; p=4.443 \times 10^{-10}\right)$, no significant difference observed between block 5 and other blocks except for block 1 ( $p=0.001$ with Bonferroni correction). Approximately $10-20 \%$ performance improvement was observed. No significant correlation was observed between the change in the Glu/GABA ratio and performance improvement $\left(r_{(13)}=-0.09, p=0.773\right)$ in non-specific learning (Fig. 9B).

In non-specific learning, no significant effect was observed in the linear increment in preparation-related activity in right M1 (T-score $=1.91$, un-corrected $p=0.080$ ). 
No significant correlation was observed between changes in the Glu/GABA ratio and M1 seed-based resting-state functional connectivity changes in the FPN $\left(r_{(13)}=-0.32, p=0.291\right)$ and $\operatorname{SMN}\left(r_{(13)}=0.15, p\right.$ $=0.616$ ) after non-specific learning (Fig. 10).

\section{Discussion}

In this study, MRS, task fMRI, and resting-state fMRI methods were combined to assess network-level changes during motor sequence learning using a 7T MR machine. This study replicated and extended previous findings regarding the crucial role of M1 in motor sequence learning. To the best of our knowledge, this is the first report to demonstrate that the local excitatory-inhibitory balance within M1 regulates $\mathrm{M} 1$ connectivity with the FPN.

GABA and Glu measurements were of high quality and reproducibility (Table 1). Although the neural excitatory-inhibitory balance is crucial for learning and memory, the main focus of the prior studies on motor learning has been limited to the evaluation of $\operatorname{GABA}^{16,18,19,23,24}$ due to technical limitations. The subtraction of two independent spectra to remove the overlap of signals is required in 3T MRS. Conversely, 7T MRS is able to concurrently resolve GABA, Glu, and glutamine (GIn), as sensitivity and chemical shift dispersion increase with increasing magnetic field strength ${ }^{25}$. A higher SNR that increases linearly with the magnetic field strength enables more accurate detection of weak signals from neurotransmitters in smaller voxels and with shorter measurement times ${ }^{26}$. Neurotransmitters were measured within M1 using a voxel size of $8 \mathrm{~cm}^{3}(2 \times 2 \times 2)$ at 7T in this study; however, a voxel size of 27 $\mathrm{cm}^{3}(3 \times 3 \times 3)$ was selected at $3 T^{27,28}$. The $3 T$ MRS is relatively insensitive to subtle changes in neurotransmitters underscoring cognitive functions due to large MRS voxel sizes ${ }^{29}$. Thus, a 7T MRS is advantageous over a 3T MRS in terms of observing neurotransmitter function in a specifically localized brain region related to changes in cognitive and behavioral task performance.

We also measured GABA and Glu levels within M1 at pre-training and post-training resting-state conditions, and during task execution using a 7T MR system. We found significant reductions in Glu ( $p<$ 0.05 , repeated-measures ANOVA and t-test with Bonferroni correction) between pre-task and post-task periods and between during-task and post-task periods (Fig. 2B). The decrease in Glu probably reflects the decrease in synaptic Glu or glutamatergic cycling as a part of energy metabolism in the tricarboxylic acid cycle ${ }^{30}$. These findings point to a learning-related decrease in Glu within M1 in motor sequence learning. A previous study using 7T machine showed no change in Glu within M1 during motor sequence learning using a serial reaction time task ${ }^{17}$. It is important to point out that, instead of implicit learning which mainly involved in the $\mathrm{M}^{31}{ }^{31}$, we adopted the explicit motor sequence learning, which is known to recruit global brain network ${ }^{9,32}$. The motor engram was shown to be generated in the parietal regions distant from M1 during the explicit learning with the instruction of "tap the sequence as fast and correct as possible" (maximum mode), whereas generated in the M1 and dorsal premotor cortex during the implicit learning through visually guided constant speed execution (constant mode) ${ }^{9}$. Glu is known to exhibit a global effect on the BOLD response via glutamatergic projections to other cortical regions 
rather than modulating the BOLD response within the acquired MRS voxel ${ }^{33,34}$. Based on these findings, the decrease in Glu is assumed to likely be related to the sequence learning-specific recruitment of the global brain network.

With regard to GABA, although previous studies showed GABA reduction in M1 during motor learning ${ }^{17,23}$, no significant difference in GABA (Fig. 2B). Our results are in line with the recent study using similar motor sequential tasks ${ }^{19}$. The GABA measured using the MRS thought to reflect bulk GABA from a large volume of interest, and is thought to reflect cellular, rather than synaptic GABA levels predominantly 24,35 . No correlation was observed between GABA with MRS and phasic GABA signaling using TMS ${ }^{36,37}$. Although a significant correlation between GABA with MRS and tonic GABA was observed in one study ${ }^{36}$, no correlation was observed in a recent study ${ }^{37}$. The main factor of this difference in the two studies could be measurement methods of MRS: scan sequence (SPECIAL vs. STEAM) and magnetic field strength of the MR system (3T vs. 7T). That is, there seems to be no consensus on the relationship between the GABA with MRS and tonic GABA.

We found that GABA changes were significantly correlated with the performance improvement $(p=$ 0.018), consistent with the previous finding ${ }^{19}$. The disinhibition is to enhance Glu-related excitatory processes resulting in the decline of Glu concentration. The M1 comprises the intrinsic horizontal connection network necessary to support circuit reorganization during learning dependent on the precise balance of excitatory and inhibitory signaling within the $M 1$ networks ${ }^{1}$. We adopted the Glu/GABA ratio to account for behavioral performance changes, as the stability of cortical areas during learning depends on the balance between cortical excitation and inhibition ${ }^{38}$. Further, considering that Glu is the precursor of GABA, their concentrations are likely to be reciprocally dependent. These factors indicate that the Glu/GABA ratio corresponds to cortical excitability ${ }^{37}$ and is a more sensitive proxy for plasticity than Glu or GABA alone. We observed a positive correlation between changes in the Glu/GABA ratio and task performance improvement (Fig. 3B). This finding suggests that between-participant variation in the balance of GABA and Glu reflects improvements in motor sequence learning performance.

We observed that preparation-related activity increased linearly in fronto-parietal regions, especially in the right M1 (Fig. 5B). This result is consistent with that of our previous study ${ }^{39}$. In explicit motor sequence learning, participants needed to retrieve whole-sequence information at the preparation phases internally. Electrophysiological studies in nonhuman primates demonstrated an increase in neuronal responses reflecting preparatory activity for movement in $\mathrm{M} 1$ as learning progressed ${ }^{40}$. Thus, the increase in preparation-related activity represents motor learning as an ecphoric process without being confounded by motor execution effects dependent on speed $\mathrm{d}^{41-43}$ and force ${ }^{44}$. The motor learning-related information of the specific sequence was accumulated in M1 because no such significant effect was observed in the M1 of the control group which conducted the sequential finger tapping with 120 different sequences. In addition, an increment of the preparatory activity was highly present in regions included SMN and FPN, thereby suggesting that the learning-related information is distributed in networks associated with both motor and executive controls. 
We also assessed resting-state $\mathrm{M} 1$ seed-based functional connectivity change elicited by motor sequence learning. As shown in Figure $6 \mathrm{C}$, a positive correlation was observed between changes in the Glu/GABA ratio within $\mathrm{M} 1$ and $\mathrm{M} 1$ seed-based resting-state functional connectivity changes in FPN. In contrast, no correlation was found in the SMN. These results reflect the learning effect during motor sequence learning because no such correlation was observed in the control group (Fig. 10). The FPN controls coordinated behavior in a rapid, accurate, and flexible goal-driven manner ${ }^{12}$. Therefore, this finding indicates that motor learning driven by cognitive control is associated with local changes in excitatoryinhibitory balance in the M1. As described above, these findings reflect individual differences in skills, effort, and concentration of self-paced movement because participants were required to execute the task as quickly as possible during learning.

To further probe the relationship between M1 and FPN, we assessed the correlations of connectivity changes in the bilateral PFC and PPC with changes in the Glu/GABA ratio of the M1. These correlations were more prominent in parietal regions than in frontal regions, thereby suggesting that the Glu/GABA ratio of the M1 is more likely to affect the connectivity with the PPC in FPN (Fig. 7). This finding concurs with the notion that the PPC is necessary for early and late learning phases, whereas the PFC is primarily involved in early learning phases ${ }^{45}$. The PFC processes sensory inputs, motor outputs, and working memory ${ }^{46-48}$. The PPC, encompassing the IPL and SPL, processes spatial-sequential components ${ }^{31,49}$. Both the M1 and PPC are critical hubs for the late motor sequence learning phase because these areas contribute to the delayed recall of learned motor sequences ${ }^{50,51}$. In other words, in the later phase of learning, PPC and M1 are involved in retrieving the learned sequences acquired during the early learning phase. Our results, combined with our previous data, suggest that M1 integrates the accumulated information processed by the PPC in motor sequence learning.

These findings are consistent with those of Sami et al. ${ }^{52}$, who investigated the consolidation effects on the resting state network using dual regression Independent Component Analysis (ICA) analysis following implicit and explicit learning, with serial reaction time task. The authors had demonstrated the role of FPN in the explicit learning group, six hours following the initial acquisition, and have interpreted this finding as bringing the learned sequence back to declarative awareness. Furthermore, they directly compared explicit and implicit groups at this late state, thereby identifying bilateral activation in both the parietal and premotor regions. The authors also speculated that this network might represent an engram of the extra procedural learning skill that had developed in the explicit acquisition group ${ }^{52}$. Therefore, we conclude that the M1 centered network with FPN represents the formation of declarative procedural skills.

As represented in Figure 4, we observed similar spatial patterns of activity in the execution and preparation phases. These areas represent the large-scale functional motor network, necessary for performing sequential motor tasks. The selection of a particular motor sequence is based on inputs from the prefrontal cortex and parietal-temporal regions to the ventral premotor cortex $(\mathrm{PMv})^{53,54}$. The dorsal part of the IPL (dIPL) is a multimodal sensory association region involved in the initial acquisition and learning of a motor task. The anterior parts of the IPL, PMv, and M1 consist of the fine motor control 
network $^{54-57}$. The dorsal premotor cortex (PMd) is involved in movement selection ${ }^{58}$. In addition, preparation-related activity was most prominently associated with enhanced activity in the putamen (Fig. $4 B)$, suggesting that this preparatory activity represents preceding self-initiated movements ${ }^{59}$. Our findings are consistent with previous results demonstrating preparatory activity in the motor, somatosensory, parietal, and prefrontal cortical regions, basal ganglia, and cerebellum in sequential finger movements ${ }^{60}$.

The participants recruited in this study were predominantly women, with body weights of $60 \mathrm{~kg}$ or less. This limitation contributed to technical challenges in MRS measurements using a single-transmit 7T MR system. First, the B1 transmit field inhomogeneity was enhanced. The suppression of water signals for the measurement of metabolites may have been insufficient depending on the head size, and it was challenging to obtain good spectral quality. Second, adjustments of MRS sequence parameters may have been necessary, involving a lengthening of measurement time to solve the local specific absorption rate limitations partly defined using body weight. Gender differences are known to affect visuo-motor adaptation learning of throwing ${ }^{61}$; given that the participants in this study were primarily women, the generalizability of the results remain limited, and further studies are warranted in which the number of men is high or at least equivalent to that of women.

In conclusion, our findings indicate that motor learning driven by cognitive control is associated with local variation in the excitatory-inhibitory balance in M1 that regulates remote connectivity with the FPN, constituting the M1-centered motor learning network.

\section{Materials And Methods}

\section{Participants}

A total of 43 healthy, right-handed adult volunteers participated in the study ( 7 male and 36 female: mean age ( \pm SD) was $22.9 \pm 4.4$ years). Handedness was assessed using the Edinburgh Handedness Inventory ${ }^{62}$. None of the participants had a history of neurological or psychiatric diseases. All participants provided written informed consent for participation in the experiment. The study was conducted according to the Declaration of Helsinki and was approved by the Ethical Committee of the National Institute for Physiological Sciences, Japan.

\section{Experimental design}

We carried out MRS-fMRI experiments using a 7T MRI scanner (MAGNETOM 7T, Siemens Healthineers, Erlangen, Germany) with a 32-channel receiving head coil and a single-channel transmitting coil (Nova Medical Inc., MA, USA). All participants underwent resting-state fMRI and MRS scans before and after the motor sequence learning tasks, as well as one MRS and four $\mathrm{fMRI}$ scans during motor sequence learning tasks in the task session (Fig. 1A). Dielectric pads $(\mathrm{CaTiO})^{63}$ were placed around each participant's head 
while scanning at $7 \mathrm{~T}$ to improve the B1 transmit field inhomogeneity. All scans were performed within the Specific Absorption Rate (SAR) limit of the normal operation mode.

\section{Motor sequence learning task}

Thirty participants were asked to perform pre-determined five-digit sequences "4-1-3-2-4" $(n=17)$ or "2-3$1-4-2 "(n=13)$ as quickly and accurately as possible in the MRI scanner (Fig. 1B) ${ }^{9-11}$. Additionally, 13 participants were asked to perform 120 different sequences to assess the non-specific learning as the control condition. The sequence "4-1-3-2-4" corresponds to "index-little-middle-ring-index." The motor sequence task consisted of six 30-s tapping epochs followed by 30-s rest epochs that were repeated five times (Fig. 1B). The visual feedback signals were displayed using a projector (Optoma EH503; Optoma Inc., Fremont, CA, USA) with a lens (APO 50-500 mm F4.5-6.3 DG OS HSM; SIGMA, Kanagawa, Japan) on a screen viewed by the participants via a mirror mounted to the receiving head coil. Response time was measured using Presentation software version 16.4 (Neurobehavioral Systems, NY, USA; RRID:

SCR_002521). The rest epoch started with the appearance of the instruction "Rest" on the screen for 500 $\mathrm{ms}$, followed by a $500-\mathrm{ms}$ presentation of four blue circles aligned within an equally spaced horizontal array. The instruction "Task" appeared for $2 \mathrm{~s}$ at the end of the rest epoch as a signal to the participants to retrieve motor sequences and prepare for their execution (Fig. 1B). The task epoch began with four closed white circles presented for $500 \mathrm{~ms}$, which changed into open circles. During the task epoch, participants tapped the button box (Current Design, Philadelphia, USA) according to the sequence shown at the top of the screen (i.e., "4-1-3-2-4"). Visual feedback of correct tapping was provided by filling the white circle corresponding to the tapped finger. When the participant provided an incorrect response, the visual feedback signal remained at the previous position until the correct button was tapped. Task performance was measured using transition time (TT), defined as the average time between two correct button responses per epoch. The performance improvement was calculated using the following equation:

Performance improvement $(\%)=\frac{\left(T T_{1}-T T_{5}\right)}{T T_{5}} \times 100$

where $\mathrm{TT}_{1}$ indicates the transition time at block 1 and $\mathrm{TT}_{5}$ indicates the transition time at block 5 . The task performance data were analyzed using repeated-measures analysis of variances (ANOVA), with block as a factor, performed using the Statistical Package for the Social Sciences software version 25.0.0 (SPSS, IBM Corp., NY, USA; RRID: SCR_002865). Two participants were excluded due to a statistical outlier in the TT values (> 2 SD).

\section{Structural data acquisition}

Three dimensional T1-weighted (T1w) images were acquired for anatomical reference (Magnetization Prepared Rapid Acquisition Gradient Echo [MPRAGE] ${ }^{64}$, TR/TE = 3,000/3.08 ms; inversion time [TI] = $1,200 \mathrm{~ms}$; field of view $=240 \times 225 \mathrm{~mm}^{2}$; matrix size $=320 \times 320$; slice thickness $=0.75 \mathrm{~mm} ; 224$ slices; 
generalized auto-calibrating partially parallel acquisitions [GRAPPA] ${ }^{65}$ acceleration factor $=3$; bandwidth $=230 \mathrm{~Hz} / \mathrm{Px}$; flip angle $=14^{\circ} ;$ acquisition time $=4 \min 50 \mathrm{~s}$ ).

\section{fMRI data acquisition}

fMRI images were acquired before, during, and after the motor sequence learning tasks using a multiband gradient-echo echo-planar imaging sequence ${ }^{66}$. The scan parameters were set as per the human connectome project (HCP) 7T protocol ${ }^{67}$ (TR/TE $=1,000 / 22.2 \mathrm{~ms}$; field of view $=208 \times 208 \mathrm{~mm}^{2}$; matrix size $=130 \times 130$; slice thickness $=1.6 \mathrm{~mm} ; 85$ slices; multi-band $/$ GRAPPA acceleration factor $=5 / 2$; bandwidth $=1,924 \mathrm{~Hz} / \mathrm{Px}$; flip angle $\left.=45^{\circ}\right)$. The spin echo field map was acquired ${ }^{68}(\mathrm{TR} / \mathrm{TE}=3,000 / 60$ ms; field of view $=208 \times 208 \mathrm{~mm}^{2}$; matrix size $=130 \times 130$; slice thickness $=1.6 \mathrm{~mm} ; 85$ slices; multiband $/$ GRAPPA acceleration factor $=5 / 2$; bandwidth $=1,924 \mathrm{~Hz} / \mathrm{Px}$; flip angle $=180^{\circ}$; acquisition time $=1$ min $26 \mathrm{~s}$ ). A B1 transmit field map in the center of the brain, around the slice of the M1 hand knob area, was acquired for each participant to optimize the input power for accurately producing a $90^{\circ}$ pulse for all fMRI scans. In particular, participants were instructed to keep their eyes open while viewing a fixation cross and to avoid having any specific thoughts or falling asleep during resting-state fMRI scans.

\section{MRS data acquisition}

A $2 \times 2 \times 2 \mathrm{~cm}^{3}$ volume of interest was centered over the right M1 hand knob area (Fig. $2 \mathrm{~A}$ ), without dura, on T1w MPRAGE images. The hand knob area was identified using fMRI during a sequential finger

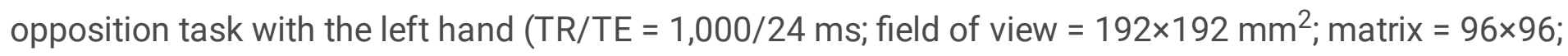
slice thickness $=2 \mathrm{~mm} ; 20$ slices; GRAPPA acceleration factor $=2$; bandwidth $=2,170 \mathrm{~Hz} / \mathrm{Px}$; flip angle $=$ $45^{\circ}$; acquisition time $=3 \mathrm{~min} 30 \mathrm{~s}$ ). Ultra-short TE MRS data were acquired before, during, and after the motor sequence learning task using the STimulated Echo Acquisition Mode (STEAM) sequence (TR/TE = $5,000 / 5.68 \mathrm{~ms}$; mixing time $=40 \mathrm{~ms}$; vector size $=2,048$; bandwidth $=4,000 \mathrm{~Hz} / \mathrm{Px}$; average $=64)$ with VAriable Power RF pulses with Optimized Relaxation delays (VAPOR) water suppression ${ }^{25,69}$. The STEAM sequence was combined with outer volume suppression to improve localization performance. A 4average water reference signal was acquired for eddy current correction and absolute quantification of the metabolites. Before data acquisition, all first- and second-order shim terms were automatically adjusted with the fast automatic shim technique using echo-planar signal readout for mapping along with projections (FASTMAP) ${ }^{70,71}$. In addition, B1 transmit field strength for localization pulses and VAPOR water suppression was adjusted for individual participants.

\section{HCP-style structural data acquisition with $3 T$ MRI and preprocessing}

In addition to the MRS-fMRI data acquisition using 7T MRI, the HCP-style structural data of all participants were obtained using a 3T MRI scanner (Magnetom Verio, Siemens Healthcare, Erlangen, Germany) with a 32-channel receiving head coil (Siemens Healthcare, Erlangen, Germany). The obtained 3T MRI data were utilized to correct the geometric distortion of the 7T MR data ${ }^{72}$ (see below, $F M R I$ preprocessing). Scan parameters were as per the HCP $3 \mathrm{~T}$ protocol with minor modifications ${ }^{73}$. Three- 
dimensional T1 $w$ images were acquired $\left(M_{P R A G E}{ }^{64}, \mathrm{TR} / \mathrm{TI} / \mathrm{TE}=2,400 / 1,060 / 2.24 \mathrm{~ms}\right.$; field of view = $256 \times 240 \mathrm{~mm}^{2}$; matrix size $=320 \times 320$; slice thickness $=0.8 \mathrm{~mm} ; 224$ slices; GRAPPA acceleration factor $=$ 2; bandwidth $=210 \mathrm{~Hz} / \mathrm{Px}$; flip angle $=8^{\circ}$; acquisition time $=6 \mathrm{~min} 38 \mathrm{~s}$; measurement $\left.=2\right)$. Threedimensional T2 weighted (T2w) images were acquired (Sampling Perfection with Application optimized Contrast using different angle Evolutions [SPACE] ${ }^{74}, \mathrm{TR} / \mathrm{TE}=3,200 / 560 \mathrm{~ms}$; field of view $=256 \times 240$ $\mathrm{mm}^{2}$; matrix size $=320 \times 320$; slice thickness $=0.8 \mathrm{~mm} ; 224$ slices; GRAPPA acceleration factor $=2$; bandwidth $=744 \mathrm{~Hz} / \mathrm{Px}$; turbo factor $=167$; acquisition time $=6 \mathrm{~min}$; measurement $=2$ ). All data were processed using the structural pipeline (PreFreeSurfer, FreeSurfer, and PostFreeSurfer) of the minimal HCP preprocessing pipeline version 4.0.0-alpha.5, including the following steps: gradient magnetic field nonlinearity distortion correction, T2w images to T1w image registration, and Montreal Neurologic Institute (MNI) volume registration ${ }^{73}$.

\section{MRS data analysis}

Raw MRS data were post-processed using MATLAB R2018a (The MathWorks, Inc., MA, USA; RRID: SCR_001622). Motion-corrupted data were removed to improve the spectral quality. To quantify the proportion of gray matter (GM), white matter (WM), and cerebrospinal fluid (CSF) fractions in the volume of interest, segmentation in SPM was applied to the T1w MPRAGE images. Eddy current correction and frequency correction were performed using a water reference scan, and the zero and first-order phases of the array coil were aligned using the cross-correlation method of MRspa (RRID: SCR_017292).

Subsequently, LCModel version 6.3-1N ${ }^{75,76}$ (Stephen Provencher, Inc., ON, Canada; RRID: SCR_014455) analysis was used to quantify the concentration of neurochemicals within the chemical shift range of 0.5 to $4.1 \mathrm{ppm}^{76}$. Other parameters in the LCModel were as reported previously ${ }^{77}$. The concentrations of GABA and Glu were normalized to that of total creatine (tCr). The change in glutamate to GABA ratio (Glu/GABA) after the motor sequence learning task was calculated using the following equation:

\section{Glu/GABA change $(\%)=\frac{\left(\mathrm{Glu} / \mathrm{GABA}_{\text {post }}-\mathrm{Glu} / \mathrm{GABA}_{\text {pre }}\right)}{\mathrm{Glu} / \mathrm{GABA}_{\text {pre }}} \times 100$}

where Glu/GABApre and Glu/GABApost indicate the Glu/GABA ratio at pre-task and post-task, respectively. The distribution of GABA and Glu concentrations was visualized using the RainCloudPlots Python-script $^{78}$ (https://github.com/RainCloudPlots/RainCloudPlots). A repeated-measures ANOVA was carried out in SPSS, with the concentrations of GABA and Glu at different time points (pre-task, duringtask, and post-task) as a factor. The Cramer-Rao lower bounds (CRLB), water linewidth at FWHM, and signal-to-noise ratio (SNR) were used for the quality control of spectra ${ }^{76}$. The CRLB and SNR were calculated using LCModel, and water linewidth was obtained by fitting to the additional water spectrum using MATLAB. Data were excluded when CRLB $>15 \%(n=1)$, linewidth $>19 \mathrm{~Hz}(n=1)$, or SNR $<30$. A 
repeated-measures ANOVA was performed on the CRLB, water linewidth, and SNR time points (pre-task, during-task, and post-task) with a within-subjects factor using SPSS.

\section{fMRI preprocessing}

All fMRI data were processed using the functional pipeline (fMRIVolume) of the minimal HCP preprocessing pipeline ${ }^{72}$. This pipeline included the following steps: motion correction, gradient magnetic field nonlinearity distortion correction, field map-based distortion correction (Topup) ${ }^{68}$, nonlinear registration into 3T MNI structure data, and grand-mean intensity normalization. Finally, volume-based smoothing with a 5-mm full width at half maximum (FWHM) Gaussian kernel was applied.

\section{Task fMRI data analysis}

Task fMRI data analysis was performed using Statistical Parametric Mapping (SPM12; RRID: SCR_007037) in MATLAB R2018a. A general linear model (GLM) was fitted to the fMRI data for each participant ${ }^{79,80}$. The fMRI time series for preparation phases $2 \mathrm{~s}$ before task execution and execution phases were modeled with boxcar functions convolved with the canonical hemodynamic response function. Each block consisted of six execution-related and preparation-related regressors. The design orthogonality between the execution and preparation phases was $-0.0137 \pm 0.054$ for block $1,-0.0141 \pm$ 0.054 for block $2,-0.0137 \pm 0.054$ for block 3 , and $-0.0139 \pm 0.054$ for block 4 (mean \pm SD). Temporal high-pass filtering with a cutoff frequency of $1 / 128 \mathrm{~Hz}$ was applied. Using a first-order autoregressive model, the serial autocorrelation was estimated from the pooled active voxels with the restricted maximum likelihood procedure and subsequently used to whiten the data ${ }^{81}$. Several nuisance covariates, including six head motion parameters and CSF time-series, were incorporated into the model. The parameter estimates for each execution-related and preparation-related regressors were assessed using constant and predefined linear contrasts. Increasing contrast vectors were defined numerically as an increment of one per block, maintaining the mean equal to zero.

For group-level analysis of task fMRI data, one-sample t-tests of participants' contrast images were performed $^{82}$. The resulting set of voxel values for each contrast constituted the SPM $\{t\}$. We calculated the T-score of linear increment in preparation-related activity in right M1 in non-specific learning. The statistical threshold was set at $p<0.05$, FWE-corrected at the voxel-level ${ }^{83}$, unless otherwise specified.

\section{Anatomical labeling and visualization}

MRIcron (RRID: SCR_008264) was used to display fMRI activation maps on a standard brain image. The Automated Anatomical Labeling atlas was used for anatomical labeling ${ }^{84}$.

\section{Resting-state fMRI data analysis}

Resting-state functional connectivity analysis was conducted using the CONN toolbox version 17 in SPM12 ${ }^{85}$ (RRID: SCR_009550). An anatomical component-based noise correction method (aCompCor) ${ }^{86}$ 
was applied to remove the five components of signals from WM, CSF, and residual head motion-related signals through linear regression. A temporal bandpass filtering of $0.008-0.090 \mathrm{~Hz}$ was applied.

Seed-to-voxel correlation analysis was performed at the individual level. We selected the preparationrelated increased voxels in $\mathrm{M} 1(\mathrm{MNI}: \mathrm{x}=36, \mathrm{y}=-25, \mathrm{z}=51)$, determined in the second-level analysis of task fMRI (FWE voxel-level corrected $p<0.05$ ), as a seed region of interest (ROI) (Fig. 6A). An individual seed-based functional connectivity map was obtained by computing Pearson's correlation coefficients between the time-series from the M1 seed ROI and the time-series of all other voxels across the whole brain. Fisher's r-to- $z$ transformation was used to convert the correlation coefficients into $z$-scores. M1seeded functional connectivity changes were integrated using the following equation using AFNI version 18.1.32. (RRID: SCR_005927):

\section{Connectivity change $=\sum\left(\right.$ Connectivity $_{\text {post }}-$ Connectivity $\left._{\text {pre }}\right)$}

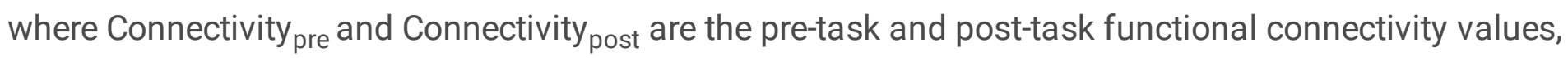
respectively.

We calculated the changes in functional connectivity within ROIs of the SMN and FPN defined from CONN's ICA analyses of the HCP dataset of 497 individuals. The SMN includes the supplementary motor cortex and bilateral sensorimotor cortex, whereas the FPN consists of the bilateral LPFC and PPC. The correlations between Glu/GABA changes within M1 and M1 seed-based functional connectivity changes were analyzed using linear regression analysis.

\section{Declarations}

\section{Data availability}

The datasets generated in this study are available from the corresponding author on reasonable request.

\section{Acknowledgments}

We would like to thank Dr. Małgorzata Marjańska, Dr. Edward J. Auerbach, Dr. Essa Yacoub, Dr. Steen Moeller (Center for Magnetic Resonance Research, University of Minnesota) for providing the MRS and fMRI sequences, and Dr. Hans-Peter Fautz, Dr. Tobias Kober, Dr. Tim DeVito, and Dr. Josef Pfeuffer (Siemens Healthineers $\mathrm{GmbH}$ ) for providing the sequences of pre-scanning adjustment on 7T MRI. This work was supported in part by the Japan Agency for Medical Research and Development (AMED) under Grant Number JP18dm0107152 and JP18dm0307005, and by the Japan Society for the Promotion of Science (JSPS) under Grant Number 20J13798 (S.M.), 16H03305 (M.F.), and 19K22985 (M.F.).

\section{Author Contributions}


S.M. designed the experiments, conducted the experiments, analyzed the experimental data, and wrote the manuscript. M.F. designed and conducted the experiments. S.K.S. helped with the experimental design and data analysis. Y.H.H helped with data analysis. T.Y. contributed analysis tools. N.S. supervised the overall project and edited the manuscript.

Competing interests: The authors declare that they have no competing interests.

\section{References}

1. Sanes, J. N. \& Donoghue, J. P. Plasticity and primary motor cortex. Annu. Rev. Neurosci.23, 393-415 (2000).

2. Shmuelof, L., Krakauer, J. W. \& Mazzoni, P. How is a motor skill learned? Change and invariance at the levels of task success and trajectory control. J. Neurophysiol.108, 578-594 (2012).

3. Miller, G. A., Galanter, E. \& Pribram, K. H. Plans and the structure of behavior. (Henry Holt, 1960).

4. Guadagnoli, M. A. \& Lee, T. D. Challenge point: A framework for conceptualizing the effects of various practice conditions in motor learning. J. Mot. Behav.36, 212-224 (2004).

5. Wadden, K. P., Hodges, N. J., De Asis, K. L., Neva, J. L. \& Boyd, L. A. Individualized challenge point practice as a method to aid motor sequence learning. J. Mot. Behav.51, 467-485 (2019).

6. Debas, K. et al. Brain plasticity related to the consolidation of motor sequence learning and motor adaptation. Proc. Natl. Acad. Sci. U. S. A.107, 17839-17844 (2010).

7. Fischer, S., Hallschmid, M., Elsner, A. L. \& Born, J. Sleep forms memory for finger skills. Proc. Natl. Acad. Sci. U. S. A.99, 11987-11991 (2002).

8. Fischer, S., Nitschke, M. F., Melchert, U. H., Erdmann, C. \& Born, J. Motor memory consolidation in sleep shapes more effective neuronal representations. J. Neurosci.25, 11248-11255 (2005).

9. Hamano, Y. H., Sugawara, S. K., Yoshimoto, T. \& Sadato, N. The motor engram as a dynamic change of the cortical network during early sequence learning: An fMRI study. Neurosci. Res.153, 27-39 (2020).

10. Walker, M. P., Brakefield, T., Morgan, A., Hobson, J. A. \& Stickgold, R. Practice with sleep makes perfect: Sleep-dependent motor skill learning. Neuron35, 205-211 (2002).

11. Walker, M. P., Brakefield, T., Hobson, J. A. \& Stickgold, R. Dissociable stages of human memory consolidation and reconsolidation. Nature425, 616-620 (2003).

12. Marek, S. \& Dosenbach, N. U. F. The frontoparietal network: Function, electrophysiology, and importance of individual precision mapping. Dialogues Clin. Neurosci.20, 133-140 (2018).

13. Dupont-Hadwen, J., Bestmann, S. \& Stagg, C. J. Motor training modulates intracortical inhibitory dynamics in motor cortex during movement preparation. Brain Stimul.12, 300-308 (2019).

14. Shmuelof, L. \& Krakauer, J. W. Are we ready for a natural history of motor learning? Neuron72, 469476 (2011).

15. Dayan, E. \& Cohen, L. G. Neuroplasticity subserving motor skill learning. Neuron72, 443-454 (2011). 
16. Stagg, C. J., Bachtiar, V. \& Johansen-Berg, H. The role of GABA in human motor learning. Curr. Biol.21, 480-484 (2011).

17. Kolasinski, J. et al. The dynamics of cortical GABA in human motor learning. J. Physiol.597, 271282 (2019).

18. Sampaio-Baptista, C. et al. Changes in functional connectivity and GABA levels with long-term motor learning. Neuroimage106, 15-20 (2015).

19. King, B. R. et al. Baseline sensorimotor GABA levels shape neuroplastic processes induced by motor learning in older adults. Hum. Brain Mapp.41, 3680-3695 (2020).

20. Vincent, J. L., Kahn, I., Snyder, A. Z., Raichle, M. E. \& Buckner, R. L. Evidence for a frontoparietal control system revealed by intrinsic functional connectivity. J. Neurophysiol.100, 3328-3342 (2008).

21. Steele, P. M. \& Mauk, M. D. Inhibitory control of LTP and LTD: Stability of synapse strength. J. Neurophysiol.81, 1559-1566 (1999).

22. Riedel, G., Platt, B. \& Micheau, J. Glutamate receptor function in learning and memory. Behav. Brain Res.140, 1-47 (2003).

23. Floyer-Lea, A., Wylezinska, M., Kincses, T. \& Matthews, P. M. Rapid modulation of GABA concentration in human sensorimotor cortex during motor learning. J. Neurophysiol.95, 1639-1644 (2006).

24. Stagg, C. J. et al. Local GABA concentration is related to network-level resting functional connectivity. Elife3, e01465 (2014).

25. Tkáč, I., Öz, G., Adriany, G., Uğurbil, K. \& Gruetter, R. In vivo 1H NMR spectroscopy of the human brain at high magnetic fields: Metabolite quantification at 4T vs. 7T. Magn. Reson. Med.62, 868-879 (2009).

26. Terpstra, M. et al. Test-retest reproducibility of neurochemical profiles with short-echo, single-voxel MR spectroscopy at 3T and 7T. Magn. Reson. Med.76, 1083-1091 (2016).

27. Greenhouse, I., King, M., Noah, S., Maddock, R. J. \& Ivry, R. B. Individual differences in resting corticospinal excitability are correlated with reaction time and GABA content in motor cortex. J. Neurosci.37, 2686-2696 (2017).

28. Sanaei Nezhad, F. et al. Number of subjects required in common study designs for functional GABA magnetic resonance spectroscopy in the human brain at 3 Tesla. Eur. J. Neurosci.51, 1784-1793 (2020).

29. Talsma, L., Loon, A. van, Scholte, H. S. \& Slagter, H. State or trait? MRS-measured GABA and Glutamate concentrations are not modulated by task demand and do not robustly predict task performance. bioRxiv https://doi.org/10.1101/543140 (2019).

30. Ramadan, S., Lin, A. \& Stanwell, P. Glutamate and glutamine: A review of in vivo MRS in the human brain. NMR Biomed.26, 1630-1646 (2013).

31. Honda, M. et al. Dynamic cortical involvement in implicit and explicit motor sequence learning. A PET study. Brain121, 2159-2173 (1998). 
32. Sugawara, S. K., Masaki, F., Hamano, Y. H., Yoshimoto, T. \& Sadato, N. Motor engram is encoded in dormant neuronal network (Federation of European Neuroscience Societies Abstracts, 2018 FENS Forum of Neuroscience, Berlin, 2018).

33. Falkenberg, L. E., Westerhausen, R., Specht, K. \& Hugdahl, K. Resting-state glutamate level in the anterior cingulate predicts blood-oxygen level-dependent response to cognitive control. Proc. Natl. Acad. Sci. U. S. A.109, 5069-5073 (2012).

34. Duncan, N. W., Wiebking, C. \& Northoff, G. Associations of regional GABA and glutamate with intrinsic and extrinsic neural activity in humans-A review of multimodal imaging studies. Neurosci. Biobehav. Rev.47, 36-52 (2014).

35. Rae, C. D. A guide to the metabolic pathways and function of metabolites observed in human brain 1H magnetic resonance spectra. Neurochem. Res.39, 1-36 (2014).

36. Stagg, C. J. et al. Relationship between physiological measures of excitability and levels of glutamate and GABA in the human motor cortex. J. Physiol.589, 5845-5855 (2011).

37. Dyke, K. et al. Comparing GABA-dependent physiological measures of inhibition with proton magnetic resonance spectroscopy measurement of GABA using ultra-high-field MRI. Neuroimage152, 360-370 (2017).

38. Shibata, K. et al. Overlearning hyperstabilizes a skill by rapidly making neurochemical processing inhibitory-dominant. Nat. Neurosci.20, 470-475 (2017).

39. Hamano, Y. H., Sugawara, S. K., Fukunaga, M. \& Sadato, N. The integrative role of the M1 for the motor sequence learning (Society for Neuroscience Abstracts, 2019 Annual Meeting of SFN, Chicago, 2019).

40. Paz, R., Boraud, T., Natan, C., Bergman, H. \& Vaadia, E. Preparatory activity in motor cortex reflects learning of local visuomotor skills. Nat. Neurosci.6, 882-890 (2003).

41. Jäncke, L. et al. Differential magnetic resonance signal change in human sensorimotor cortex to finger movements of different rate of the dominant and subdominant hand. Brain Res. Cogn. Brain Res.6, 279-284 (1998).

42. Sadato, N. et al. Frequency-dependent changes of regional cerebral blood flow during finger movements. J. Cereb. Blood Flow Metab.16, 23-33 (1996).

43. Sadato, N. et al. Frequency-dependent changes of regional cerebral blood flow during finger movements: Functional MRI compared to PET. J. Cereb. Blood Flow Metab.17, 670-679 (1997).

44. Dettmers, C. et al. Relation between cerebral activity and force in the motor areas of the human brain. J. Neurophysiol.74, 802-815 (1995).

45. Dahms, C., Brodoehl, S., Witte, O. W. \& Klingner, C. M. The importance of different learning stages for motor sequence learning after stroke. Hum. Brain Mapp.41, 270-286 (2020).

46. Halsband, U. \& Lange, R. K. Motor learning in man: A review of functional and clinical studies. J. Physiol.99, 414-424 (2006).

47. Miller, E. K. The prefrontal cortex and cognitive control. Nat. Rev. Neurosci.1, 59-65 (2000). 
48. Miller, E. K. \& Cohen, J. D. An integrative theory of prefrontal cortex function. Annu. Rev. Neurosci.24, 167-202 (2001).

49. Jenkins, I. H., Brooks, D. J., Nixon, P. D., Frackowiak, R. S. J. \& Passingham, R. E. Motor sequence learning: A study with positron emission tomography. J. Neurosci.14, 3775-3790 (1994).

50. Doyon, J., Penhune, V. \& Ungerleider, L. G. Distinct contribution of the cortico-striatal and corticocerebellar systems to motor skill learning. Neuropsychologia41, 252-262 (2003).

51. Penhune, V. B. \& Doyon, J. Dynamic cortical and subcortical networks in learning and delayed recall of timed motor sequences. J. Neurosci.22, 1397-1406 (2002).

52. Sami, S., Robertson, E. M. \& Chris Miall, R. The time course of task-specific memory consolidation effects in resting state networks. J. Neurosci.34, 3982-3992 (2014).

53. Fagg, A. H. \& Arbib, M. A. Modeling parietal-premotor interactions in primate control of grasping. Neural Networks11, 1277-1303 (1998).

54. Rizzolatti, G. \& Luppino, G. The cortical motor system. Neuron31, 889-901 (2001).

55. Rizzolatti, G. \& Wolpert, D. M. Motor systems. Curr. Opin. Neurobiol.15, 623-625 (2005).

56. Karabanov, A. et al. Timing-dependent modulation of the posterior parietal cortex-primary motor cortex pathway by sensorimotor training. J. Neurophysiol.107, 3190-3199 (2012).

57. Merchant, S. H. I. et al. The role of the inferior parietal lobule in writer's cramp. Brain143, 1766-1779 (2020).

58. Grafton, S. T., Fagg, A. H. \& Arbib, M. A. Dorsal premotor cortex and conditional movement selection: A PET functional mapping study. J. Neurophysiol.79, 1092-1097 (1998).

59. Schultz, W. \& Romo, R. Role of primate basal ganglia and frontal cortex in the internal generation of movements - I. Preparatory activity in the anterior striatum. Exp. Brain Res.91, 363-384 (1992).

60. Nambu, I. et al. Decoding sequential finger movements from preparatory activity in higher-order motor regions: A functional magnetic resonance imaging multi-voxel pattern analysis. Eur. J. Neurosci.42, 2851-2859 (2015).

61. Moreno-Briseño, P., Díaz, R., Campos-Romo, A. \& Fernandez-Ruiz, J. Sex-related differences in motor learning and performance. Behav. Brain Funct.6, 74 (2010).

62. Oldfield, R. C. The assessment and analysis of handedness: The Edinburgh inventory. Neuropsychologia9, 97-113 (1971).

63. Webb, A. G. Dielectric materials in magnetic resonance. Concepts Magn. Reson. Part A38A, 148-184 (2011).

64. Mugler, J. P. \& Brookeman, J. R. Three-dimensional magnetization-prepared rapid gradient-echo imaging (3D MP RAGE). Magn. Reson. Med.15, 152-157 (1990).

65. Griswold, M. A. et al. Generalized autocalibrating partially parallel acquisitions (GRAPPA). Magn. Reson. Med.47, 1202-1210 (2002).

66. Moeller, S. et al. Multiband multislice GE-EPI at 7 tesla, with 16-fold acceleration using partial parallel imaging with application to high spatial and temporal whole-brain FMRI. Magn. Reson. Med.63, 
1144-1153 (2010).

67. Vu, A. T. et al. Tradeoffs in pushing the spatial resolution of fMRI for the 7T Human Connectome Project. Neuroimage154, 23-32 (2017).

68. Andersson, J. L. R., Skare, S. \& Ashburner, J. How to correct susceptibility distortions in spin-echo echo-planar images: Application to diffusion tensor imaging. Neuroimage20, 870-888 (2003).

69. Tkáč, I., Starčuk, Z., Choi, I. Y. \& Gruetter, R. In vivo 1H NMR spectroscopy of rat brain at 1 ms echo time. Magn. Reson. Med.41, 649-656 (1999).

70. Gruetter, R. Automatic, localized in Vivo adjustment of all first-and second-order shim coils. Magn. Reson. Med.29, 804-811 (1993).

71. Gruetter, R. \& Tkáč, I. Field mapping without reference scan using asymmetric echo-planar techniques. Magn. Reson. Med.43, 319-323 (2000).

72. Yamamoto, T., Fukunaga, M., Sugawara, S.K., Hamano, Y.H. \& Sadato, N. Quantitative evaluations of geometrical distortion corrections in cortical surface-based analysis of high-resolution functional MRI data at 7 tesla. J. Magn. Reson. Imaging https://doi.org/10.1002/jmri.27420 (2020).

73. Glasser, M. F. et al. The minimal preprocessing pipelines for the Human Connectome Project. Neuroimage80, 105-124 (2013).

74. Mugler, J. P. Optimized three-dimensional fast-spin-echo MRI. J. Magn. Reson. Imaging39, 745-767 (2014).

75. Provencher, S. W. Estimation of metabolite concentrations from localized in vivo proton NMR spectra. Magn. Reson. Med.30, 672-679 (1993).

76. Provencher, S. W. Automatic quantitation of localized in vivo $1 \mathrm{H}$ spectra with LCModel. NMR Biomed.14, 260-264 (2001).

77. Marjańska, M. \& Terpstra, M. Influence of fitting approaches in LCModel on MRS quantification focusing on age-specific macromolecules and the spline baseline. NMR Biomed. e4197 (2019).

78. Allen, M., Poggiali, D., Whitaker, K., Marshall, T. R. \& Kievit, R. A. Raincloud plots: A multi-platform tool for robust data visualization. Wellcome open Res.4, 63 (2019).

79. Friston, K. J., Jezzard, P. \& Turner, R. Analysis of functional MRI time-series. Hum. Brain Mapp.1, 153-171 (1994).

80. Worsley, K. J. \& Friston, K. J. Analysis of fMRI time-series revisited-again. Neuroimage2, 173-181 (1995).

81. Friston, K. J. et al. Classical and Bayesian inference in neuroimaging: Applications. Neuroimage16, 484-512 (2002).

82. Holmes, A. P. \& Friston, K. J. Generalisability, random effects \& population inference. Neuroimage7, S754 (1998).

83. Friston, K. J., Holmes, A., Poline, J. -B., Price, C. J. \& Frith, C. D. Detecting activations in pet and fMRI: Levels of inference and power. Neuroimage4, 223-235 (1996). 
84. Tzourio-Mazoyer, N. et al. Automated anatomical labeling of activations in SPM using a macroscopic anatomical parcellation of the MNI MRI single-subject brain. Neuroimage15, 273-289 (2002).

85. Whitfield-Gabrieli, S. \& Nieto-Castanon, A. Conn: A functional connectivity toolbox for correlated and anticorrelated brain networks. Brain Connect.2, 125-141 (2012).

86. Behzadi, Y., Restom, K., Liau, J. \& Liu, T. T. A component based noise correction method (CompCor) for BOLD and perfusion based fMRI. Neuroimage37, 90-101 (2007).

\section{Figures}

A

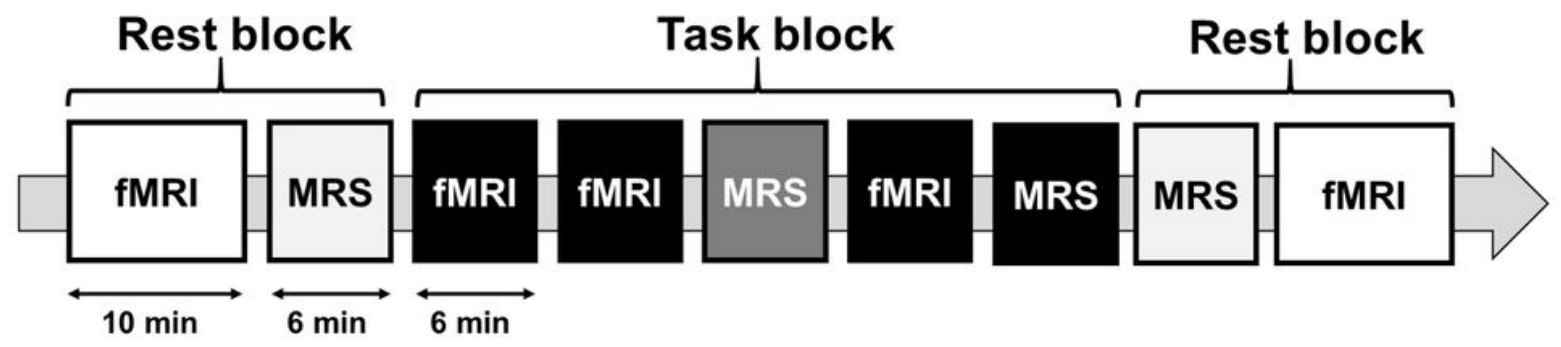

B
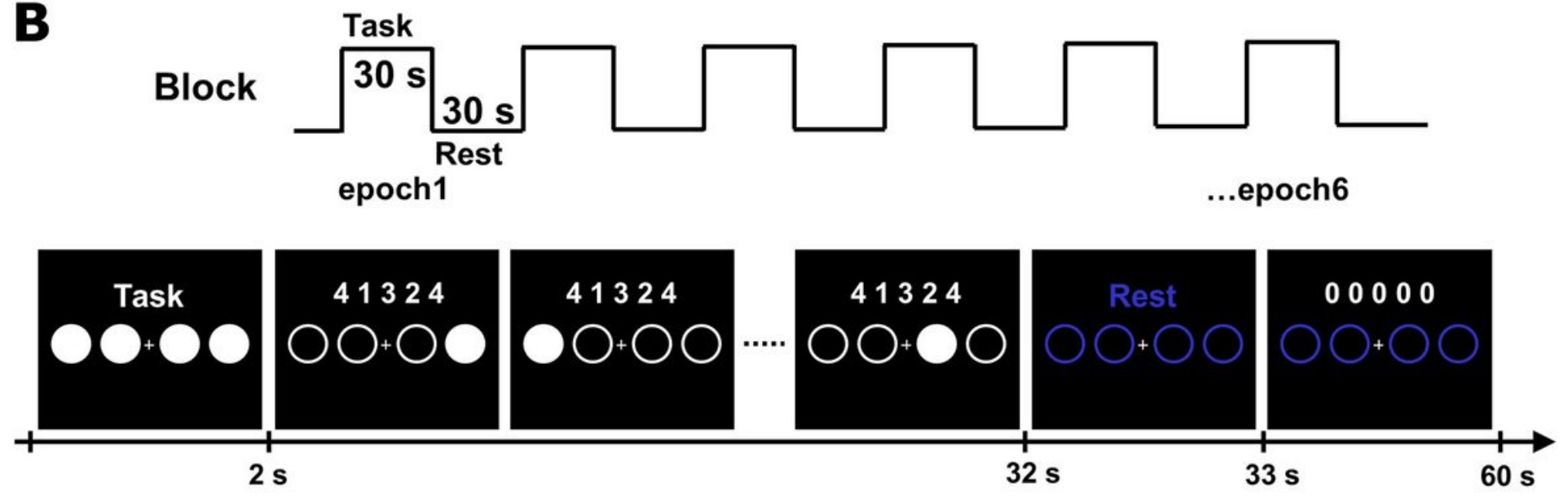

Figure 1

Experimental design (A) The timeline of combined functional magnetic resonance imaging (fMRI) and magnetic resonance spectroscopy (MRS) sessions. The experiment consists of the pre-learning rest session, followed by the task and post-learning rest session. During the rest of the sessions, fMRI and MRS scans are conducted before and after motor sequence learning. During the task session, participants undergo four fMRI scans and one MRS scan with sequential finger-tapping learning tasks. (B) Task design. Task blocks (1-5) consists of six cycles of task and rest epochs. Prior to task execution, participants are instructed to retrieve and prepare for the motor sequences following the instructions and closed circles. Participants are presented with a five-digit sequence (e.g. "4-1-3-2-4") for 30 s during the task epoch. During the rest epoch, four open blue circles were presented. 


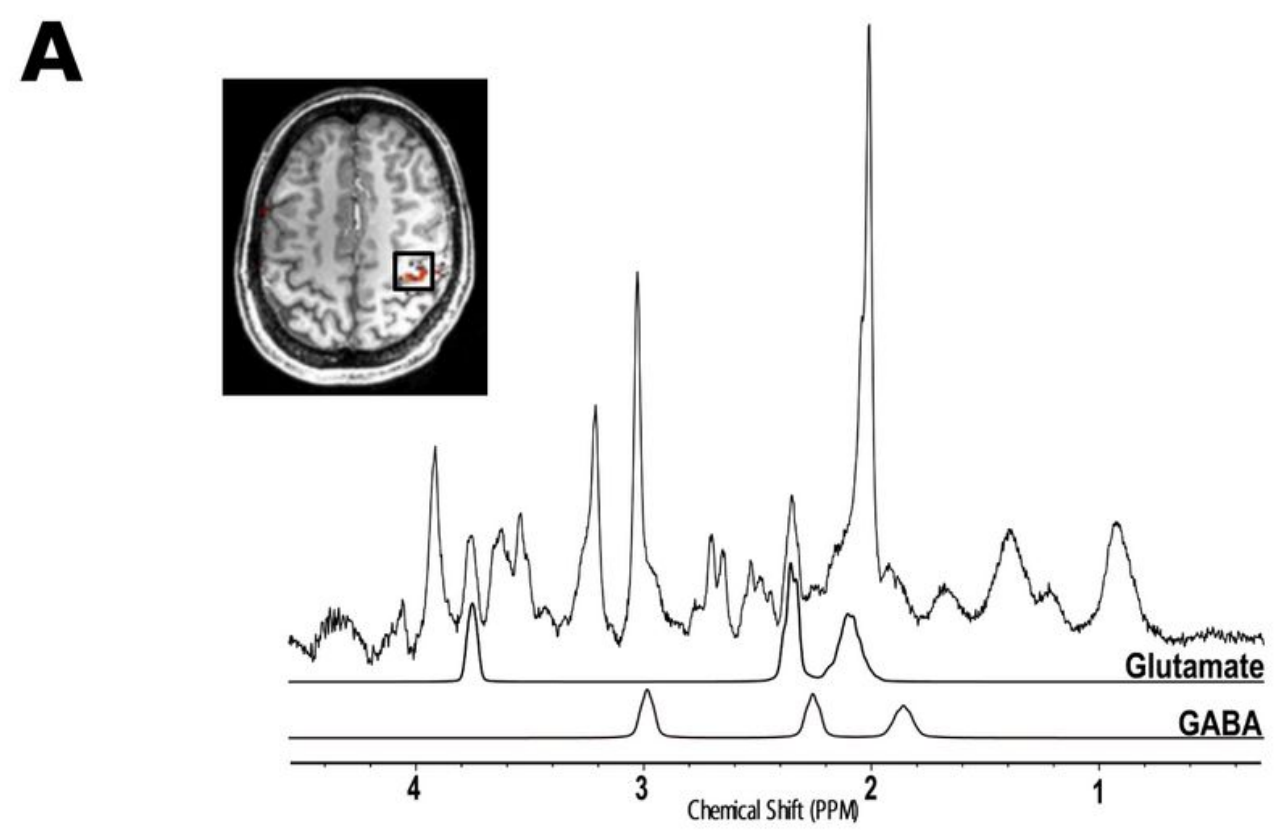

B
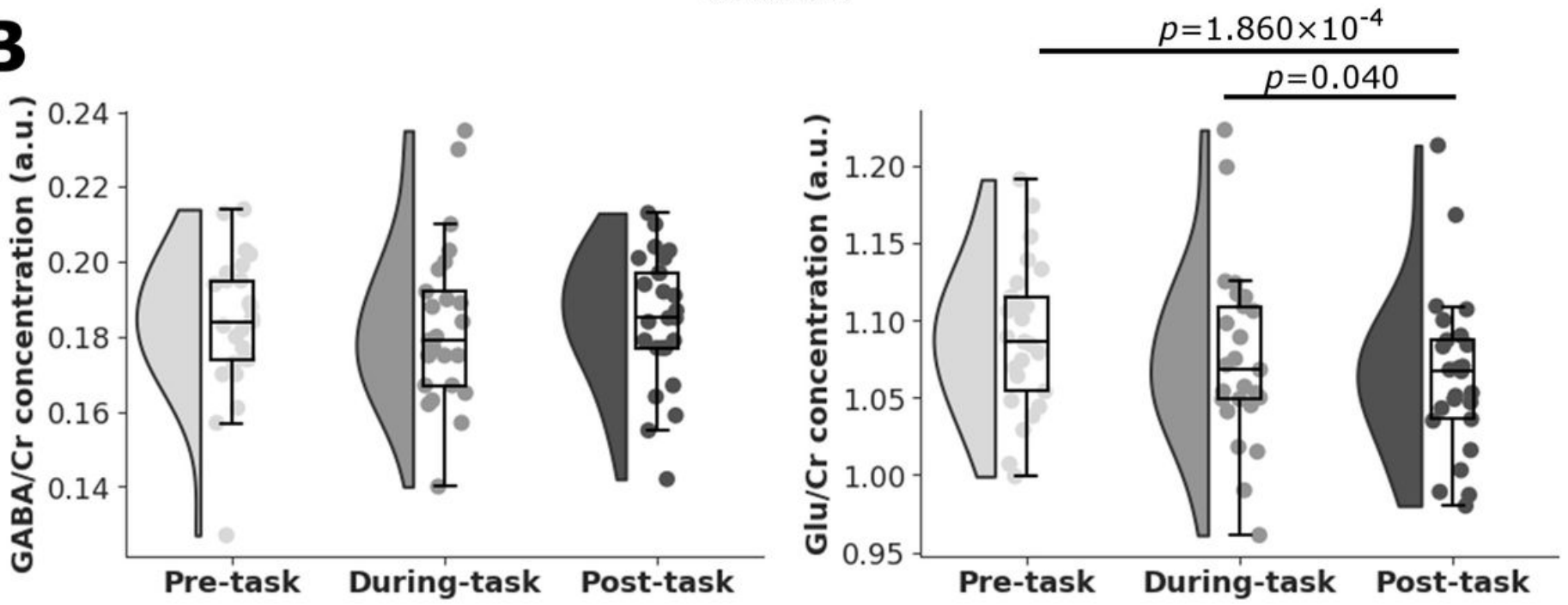

Figure 2

Magnetic resonance spectra and neurotransmitter levels (A) The $2 \times 2 \times 2 \mathrm{~cm} 3$ volume of interest (black square) is centered over the hand knob area of right primary motor cortex identified using fMRI during a finger opposition task (red) and is superimposed on the T1w MPRAGE image. (B) Violin plots coupled with boxplots showing the distribution of the concentrations of GABA and glutamate (Glu) during the pretask (light gray), during-task (dark gray), and post-task (black) periods. Each dot represents a data point $(n=25)$. The boxplots represent the median and upper/lower quartiles of the data, and the vertical lines represent the highest/lowest values. 
A

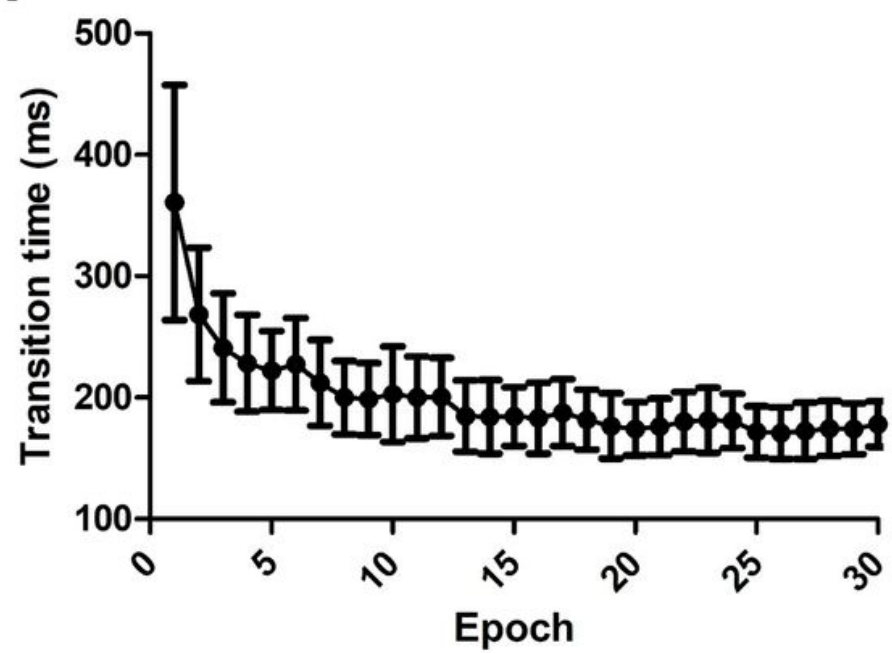

B

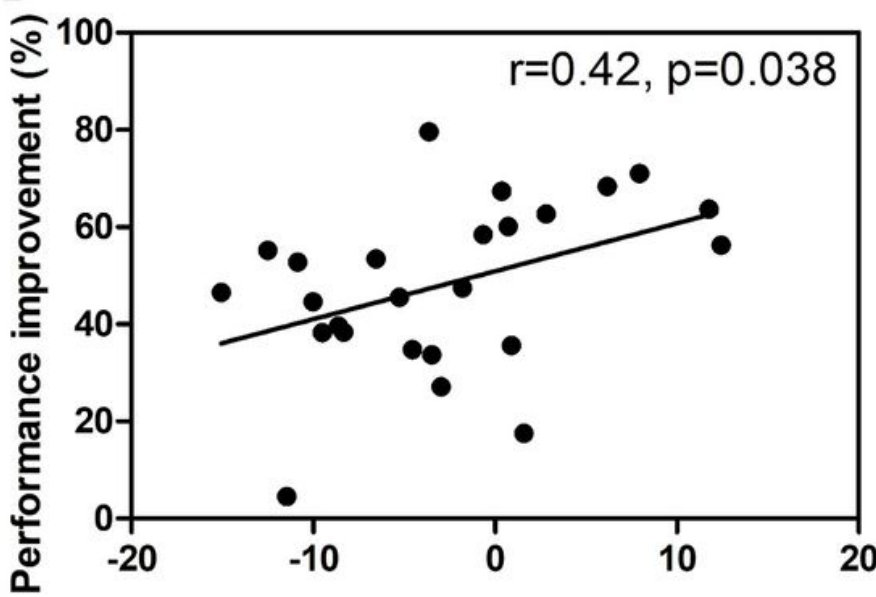

Glu/GABA change $\%$

Figure 3

Changes in Glu/GABA ratio in relation to behavioral performance improvement (A) Task performance in motor sequence learning. Task performance is measured using transition time, defined as the median time between two correct button responses per epoch. Data are presented as mean \pm standard deviation (SD) for $n=25$. (B) Relationship between Glu/GABA changes within right primary motor cortex and performance improvement.
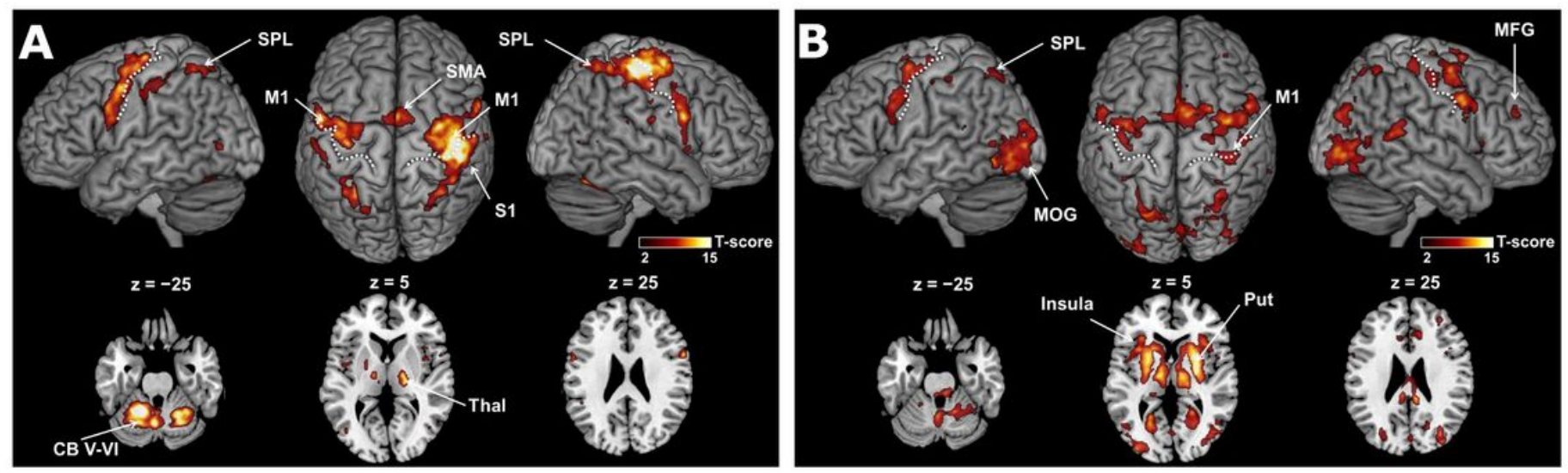

Figure 4

Constant changes during execution and preparation. (A) Execution-related and (B) preparation-related activity superimposed on the surface-rendered high-resolution magnetic resonance imaging of the template brain. The white dotted lines delineate the central sulcus. The level of statistical significance is set to $p<0.05$, family-wise error corrected for multiple comparisons at the voxel-level. CB, cerebellum; SPL, superior parietal lobule; MFG, middle frontal gyrus; MOG, middle occipital gyrus; 1 , primary 
somatosensory cortex; M1, primary motor cortex; Thal, thalamus; Put, putamen; SMA, supplementary motor area.
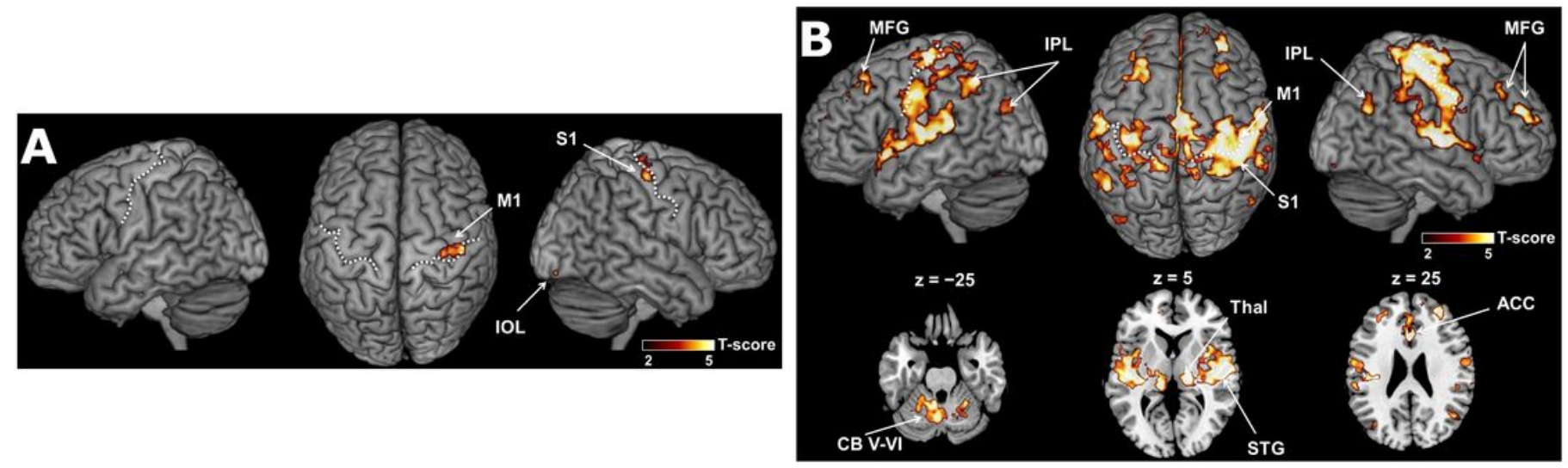

\section{Figure 5}

Learning related changes during execution and preparation. Linear increments in $(A)$ execution-related and (B) preparation-related activity superimposed on the surface-rendered high-resolution magnetic resonance imaging of the template brain. The white dotted lines indicate the central sulcus. The level of statistical significance is set to $p<0.05$, family-wise error corrected for multiple comparisons at the cluster-level. CB, cerebellum; IPL, inferior parietal lobule; MFG, middle frontal gyrus; S1, primary somatosensory cortex; M1, primary motor cortex; SMA, supplementary motor area; STG, superior temporal gyrus; Thal, thalamus; ACC, anterior cingulate cortex. 
A

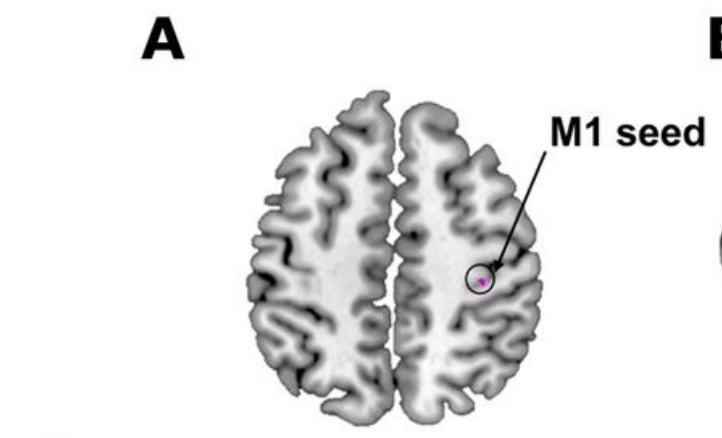

B

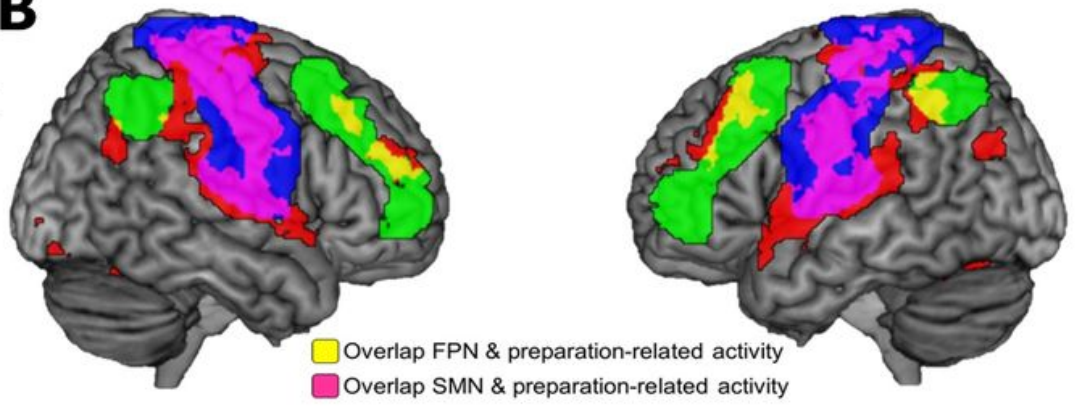

C

Sensorimotor network (SMN)

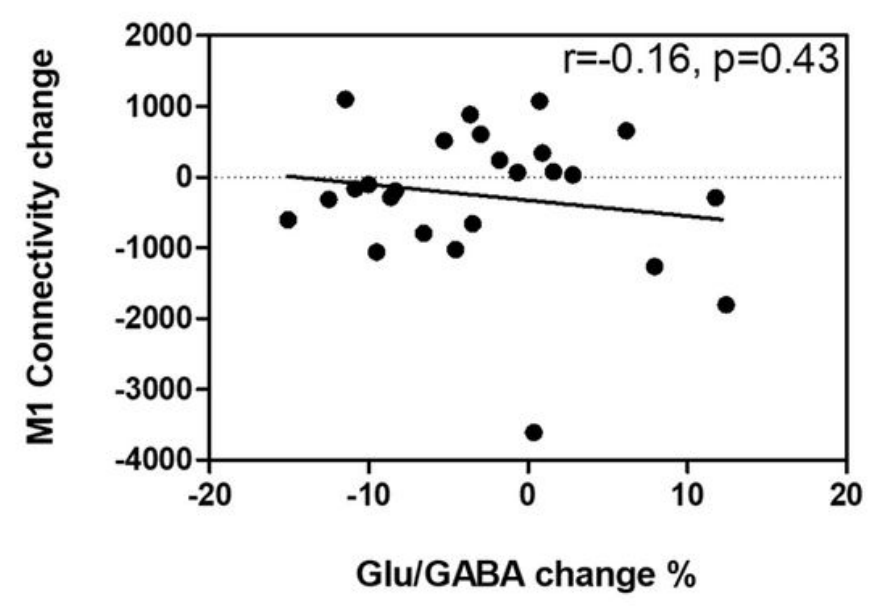

Fronto-parietal network (FPN)

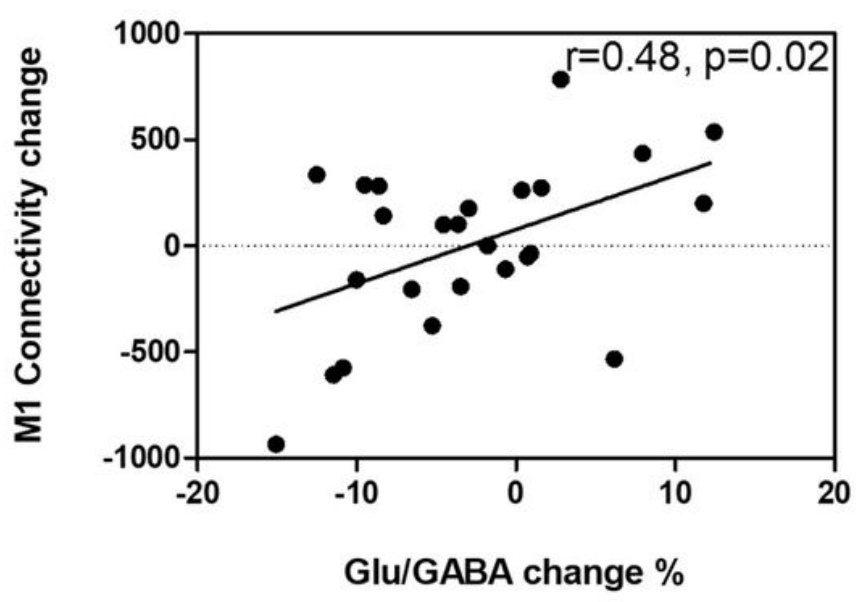

Figure 6

Region of interest (ROI)-based analysis of functional connectivity between the right primary motor cortex (M1) and fronto-parietal network (FPN) or sensorimotor network (SMN) (A) M1 seed ROI depicted by the linear increments in preparation-related activity. The level of statistical significance is set at $p<0.05$, family-wise error corrected for multiple comparisons at the voxel-level. (B) ROI overlap of SMN (blue) and FPN (green) with the learning-related network, depicted as the linear increments in preparation-related activity using task functional magnetic resonance imaging (red). SMN and FPN are defined based on the CONN toolbox. (C) Relationships between Glu/GABA changes within right M1 and M1 seed-based restingstate functional connectivity changes in the SMN and FPN after motor sequence learning. M1 seed-based resting-state functional connectivity changes were calculated from the sum of changes in connectivity values between pre-task and post-task periods in the networks. 

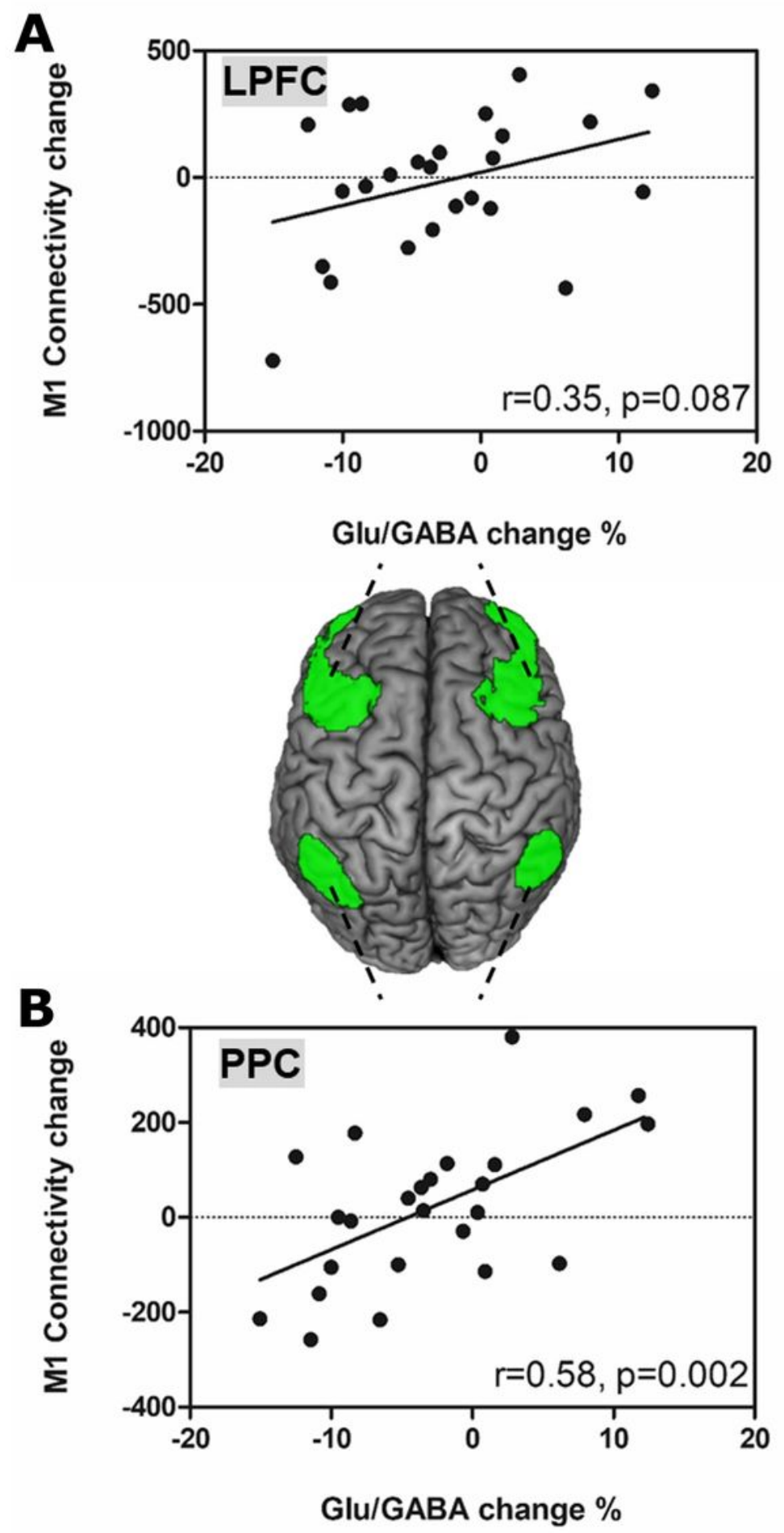

Figure 7

Region of interest-based analysis of functional connectivity between the right primary motor cortex (M1) and subregions of fronto-parietal network (FPN) Relationships between the changes in Glu/GABA ratio within the right $\mathrm{M} 1$ and $\mathrm{M} 1$ seed-based resting-state functional connectivity changes in (A) lateral prefrontal cortex (LPFC) and (B) posterior parietal cortex (PPC) of the FPN (green) after motor sequence learning. 

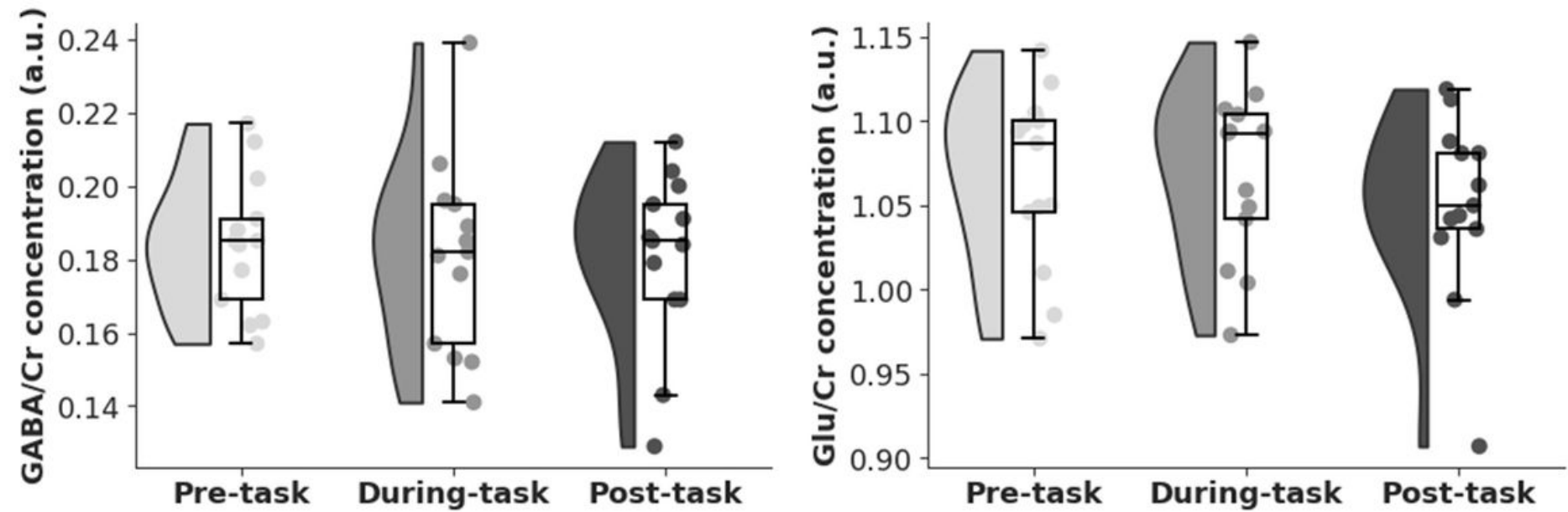

\section{Figure 8}

Neurotransmitter levels change in non-specific learning Violin plots coupled with boxplots showing the distribution of the concentrations of GABA and glutamate (Glu) during the pre-task (light gray), duringtask (dark gray), and post-task (black) periods in non-specific learning. Each dot represents a data point $(n=13)$. The boxplots represent the median and upper/lower quartiles of the data, and the vertical lines represent the highest/lowest values.
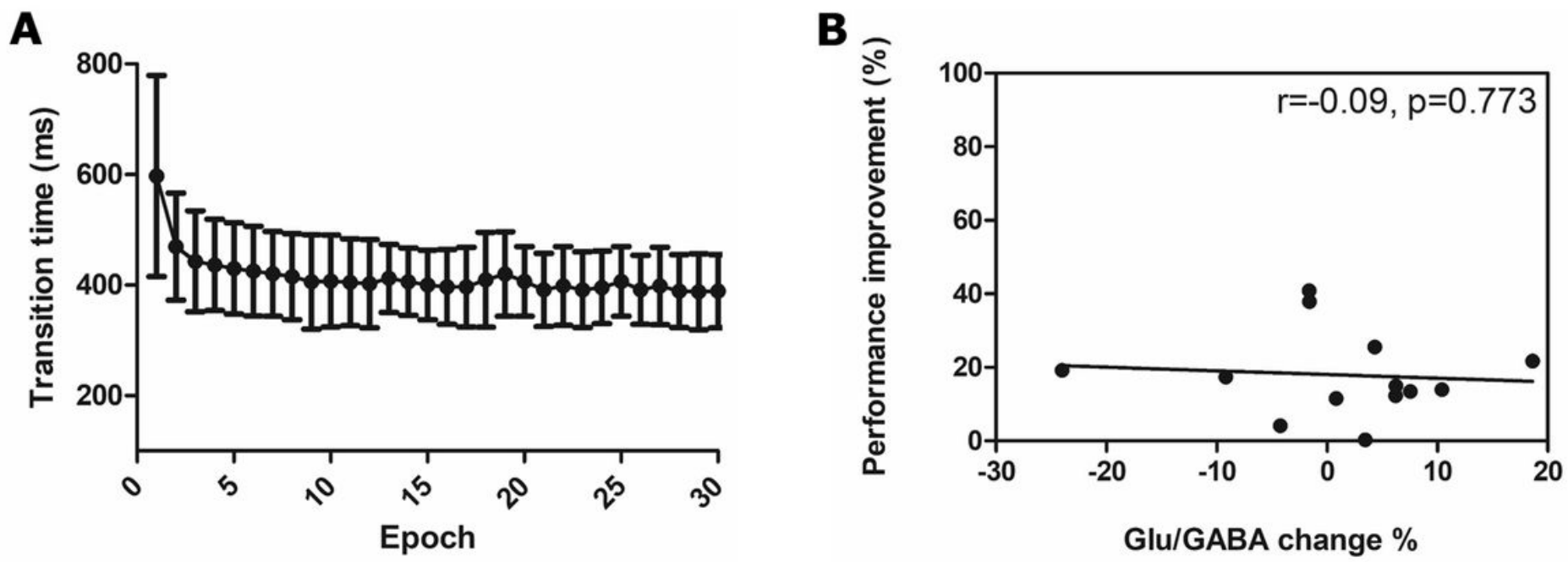

Figure 9

Changes in Glu/GABA ratio in relation to behavioral performance improvement in non-specific learning (A) Task performance in non-specific motor sequence learning. Task performance is measured using transition time, defined as the median time between two correct button responses per epoch. Data are presented as mean \pm standard deviation (SD) for $n=13$. (B) Relationship between Glu/GABA changes within right primary motor cortex and performance improvement in non-specific learning. 

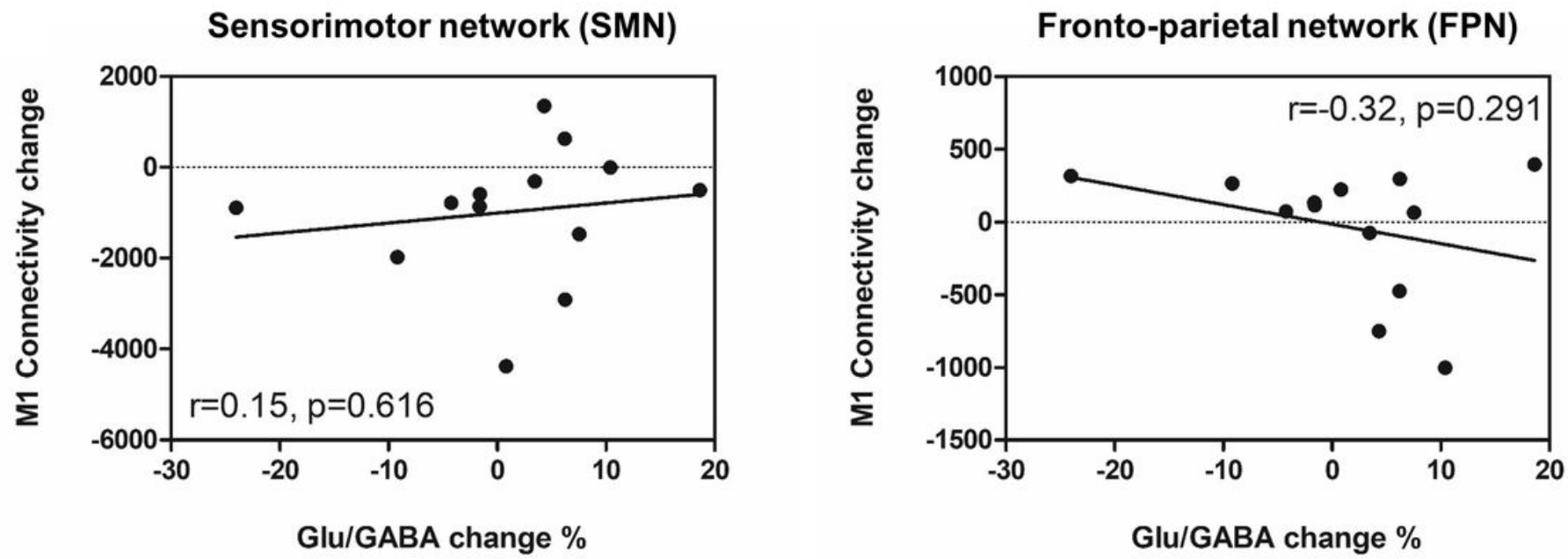

Figure 10

Region of interest-based analysis of functional connectivity between the right primary motor cortex (M1) and fronto-parietal network (FPN) or sensorimotor network (SMN) in non-specific learning Relationships between Glu/GABA changes within right M1 and M1 seed-based resting-state functional connectivity changes in the SMN and FPN after non-specific learning. M1 seed-based resting-state functional connectivity changes are calculated from the sum of changes in connectivity values between pre-task and post-task periods in the networks. 